\title{
Implantable cardiac electronic device infections: single center study
}

\author{
Grzegorz Sławiński, Maciej Kempa 니, Ewa Katarzyna Lewicka, \\ Szymon Budrejko, Tomasz Królak, Grzegorz Raczak ㅌ
}

Department of Cardiology and Electrotherapy Medical University of Gdańsk, Poland

\begin{abstract}
Implantable cardiac electronic device (ICED) infections include: lead infection (ICED-LI), device pocket infection (PI) and infective endocarditis (ICED-IE). The aim of this study is to analyze the records of patients with ICED, who developed implantable device-related infections. We analyzed retrospectively the records of the University Clinical Centre (Gdańsk) patients who in 2012-2018 underwent transvenous lead extraction (TLE) due to infections. In order to identify potential ICED infection risk factors we included patients who underwent any electrotherapy procedure within 2 years prior to the TLE. ICED infections that led to septic shock were defined as severe. The analyzed sample included 59 patients with infectious complications (37 male and 22 female) with median age of 74 . The in-hospital mortality was $8.5 \%$. All patients with severe ICED infection were diagnosed with ICED-LI, whereas the rest of the sample was diagnosed mostly with PI $(p<0.001)$. The most commonly cultured pathogens were $\mathrm{S}$. aureus and $\mathrm{S}$. epidermidis. In the analyzed sample, the most common infectious complication related to the ICED was PI and the most common etiological agents were S. aureus and S. epidermidis. Severe ICED infections that present with septic shock are associated with a $50 \%$ in-hospital mortality rate.

Keywords: infective endocarditis • septic shock • implantable cardiac electronic device infections
\end{abstract}

\section{Citation}

Sławiński G, Kempa M, Lewicka EK, Budrejko S, Królak T, Raczak G. Implantable cardiac electronic device infections: single center study. Eur J TransI Clin Med 2018; 1(1):57-62 • DOI: 10.31373/ejtcm/92167.

\section{Background}

In the recent years there was an increase in the number of implantations of implantable cardioverter-defibrillators (ICD) and pacemakers. Implantation of the above cardiac devices is a procedure with a relatively low complication rate, however if they occur the patient should be treated at a center of reference. The most serious complications, with poor long-term prognosis, are the implantable cardiac electronic device (ICED) infections which include lead infection (ICED-LI), local infec- tion in or around the device pocket (PI, pocket infection) and infective endocarditis (ICED-IE). Determining the risk factors of ICED infections is the subject of on-going research.

\section{Aim}

The aim of this study is to analyze the records of patients with ICED, who developed implantable device-related infections.

\section{Corresponding author:}

Grzegorz Sławiński, Department of Cardiology and Electrotherapy; Medical University of Gdańsk, 7 Dębinki , 80-211 Gdańsk , Poland, tel.: +48 583000000 , fax: +4858300 1000, e-mail: lek.grzegorzslawinski@gmail.com 


\section{Materials and methods}

We analyzed retrospectively the records of patients who from January 2012 to February 2018 underwent transvenous lead extraction (TLE) due to infections (ICEDLI, ICED-IE and PI) at the Department of Cardiology and Electrotherapy of the University Clinical Centre (Gdańsk). The subtypes of ICED infections were diagnosed in accordance with the current British guidelines [1]. Pocket infection was diagnosed when we noticed local features of infection without signs and symptoms of systemic infection and without positive results of blood cultures.

ICED-LI was diagnosed when we confirmed by echocardiography vegetations attached to the leads, when patient presented symptoms or signs of systemic infection. Additionally important role in the process of confirming ICED-LI constituted positive microbiological cultures and presence of Duke microbiological criteria.

ICED-IE was diagnosed when Duke criteria for infective endocarditis were met and we can confirmed vegetations attached to the valves by using echocardiography.

In accordance with the current guidelines, we have taken three blood culture samples when the patient was clinically stable or two blood culture samples when septic shock occurred. Ale these samples were taken before empiric antimicrobial therapy was started. Culture of the extracted electrode was also performed in every case during TLE.

In an attempt to identify potential risk factors of ICED infections we included records of patients who underwent any cardiac electrotherapy procedure (de novo implantation, device replacement, upgrade of the existing device) within 2 years prior to the TLE. ICED infections that led to septic shock are described as 'severe.' Septic shock was diagnosed in accordance with the Sepsis-3 guidelines [2]. Septic shock is a subset of sepsis with metabolic and circulatory abnormalities, which can be measured objectively i.e. necessity of using vasopressors to maintain MAP (mean arterial pressure) $\geq 65 \mathrm{~mm}$ $\mathrm{Hg}$ and a serum lactate level $>2 \mathrm{mmol} / \mathrm{L}(18 \mathrm{mg} / \mathrm{dL})$. We extracted the following data from patient records: demographics, previous cardiac electrotherapy procedures, ICED type, comorbidities, Charlson Comorbidity Index, laboratory parameters and pharmacotherapy prior to the ICED infection.

Charlson Comorbidity Index is a reliable measure which can be used for predicting short-term and longterm outcomes. It can be used for different patients populations. Charlson Comorbidity Index takes into consideration 19 conditions, weighted depending on their clinical significance - from one to six points (see Table 1 for details). The summation of these weighted comorbidity scores results in final Charlson Comorbidity Index [3].
Table 1. Components of Charlson Comorbidity Index

\begin{tabular}{|l|c|}
\multicolumn{1}{c|}{ Condition } & $\begin{array}{c}\text { Weight } \\
\text { [points] }\end{array}$ \\
\hline Myocardial infarction & 1 \\
\hline Congestive heart failure & 1 \\
\hline Peripheral vascular disease & 1 \\
\hline Cerebrovascular disease & 1 \\
\hline Dementia & 1 \\
\hline Chronic pulmonary disease & 1 \\
\hline Connective tissue disease & 1 \\
\hline Peptic ulcer disease & 1 \\
\hline Mild liver disease & 1 \\
\hline Diabetes without complications & 1 \\
\hline Hemiplegia & 2 \\
\hline Moderate to severe renal \\
disease
\end{tabular}

Abbreviations: AIDS - Acquired Immunodeficiency Syndrome

\section{Statistical analysis}

Statistical significance was set at $p<0,05$. The chisquared test was used for testing relationships between categorical variables. The Shapiro-Wilk test was used to test the assumption of normality and the Mann-Whitney-Wilcoxon test were applied to the comparison of two independent data whose measurements are at least ordinal. Statistical analysis was performed using the Statistica software v 12.0 .

\section{Results}

The analyzed sample included 59 patients $(37$ male and 22 female, median age 74 years, 25 th and 75 th percentile respectively 62 and 81 years of age) with ICED infections, who underwent a cardiac electrotherapy procedure within 2 years prior to the TLE (see Figure 1 for details). The indications for TLE were as follows: PI $(n=36)$, ICED-LI $(n=20)$ and ICED-IE $(n=3)$.

Severe ICED infection was diagnosed in 10 patients (17\%). We can see a trend toward significance in terms of younger age in this subset of patients comparing to the rest of the sample (median age 70 vs $74 ; p=0.09$ ). The in-hospital mortality was $8.5 \%(n=5)$ and all the deceased patients underwent severe ICED infections. All patients with severe ICED infections were diagnosed with ICED-LI $(n=10, p<0.001)$. Only $50 \%$ of patients with severe ICED infection survived. 


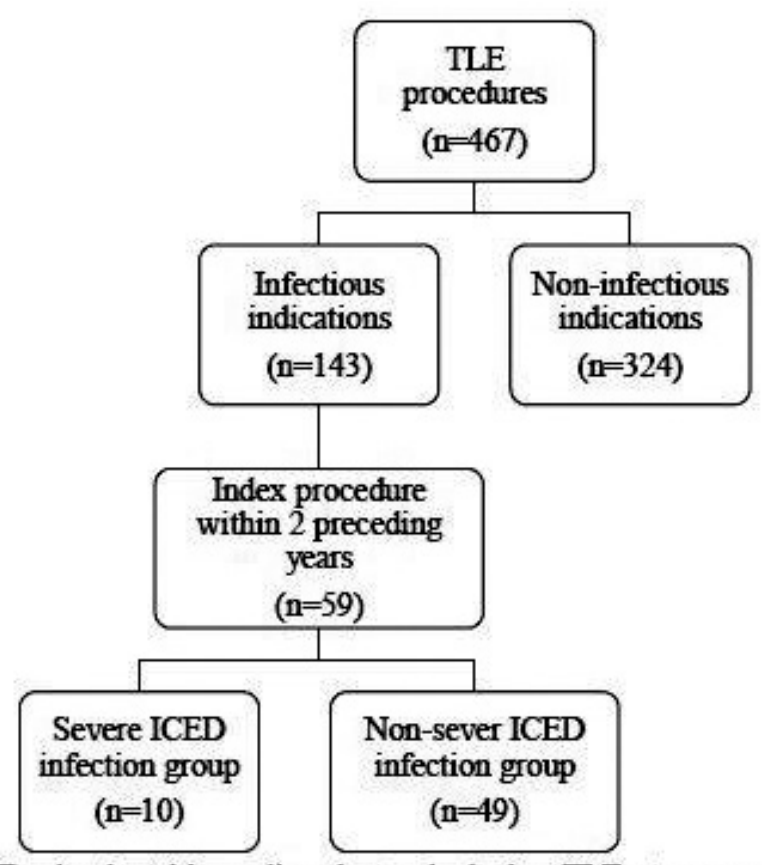

Abbreviations: ICED - implantable cardiac electronic device; TLE - transvenous lead extraction

Figure 1. Flow chart explaining the selection of eligible patients who eventually made analysed sample

$30.5 \%$ of the studied patients had positive blood cultures and the most common pathogens were $S$. aureus $(n=7)$ and $S$. epidermidis ( $n=6)$. Among the subset of patients with severe ICED infection, $50 \%$ had positive blood cultures and the pathogen was always $S$. aureus (see Figure 2). Culture of the extracted electrodes was positive in 19 (32\%) cases. Again the most common pathogens were $S$. aureus $(n=7)$ and $S$. epidermidis $(n=6)$. Other bacteria which we cultured form extracted electrodes were $S$. marcescens $(n=4), P$. aeruginosa $(n=1)$, E. faecalis $(n=1)$.

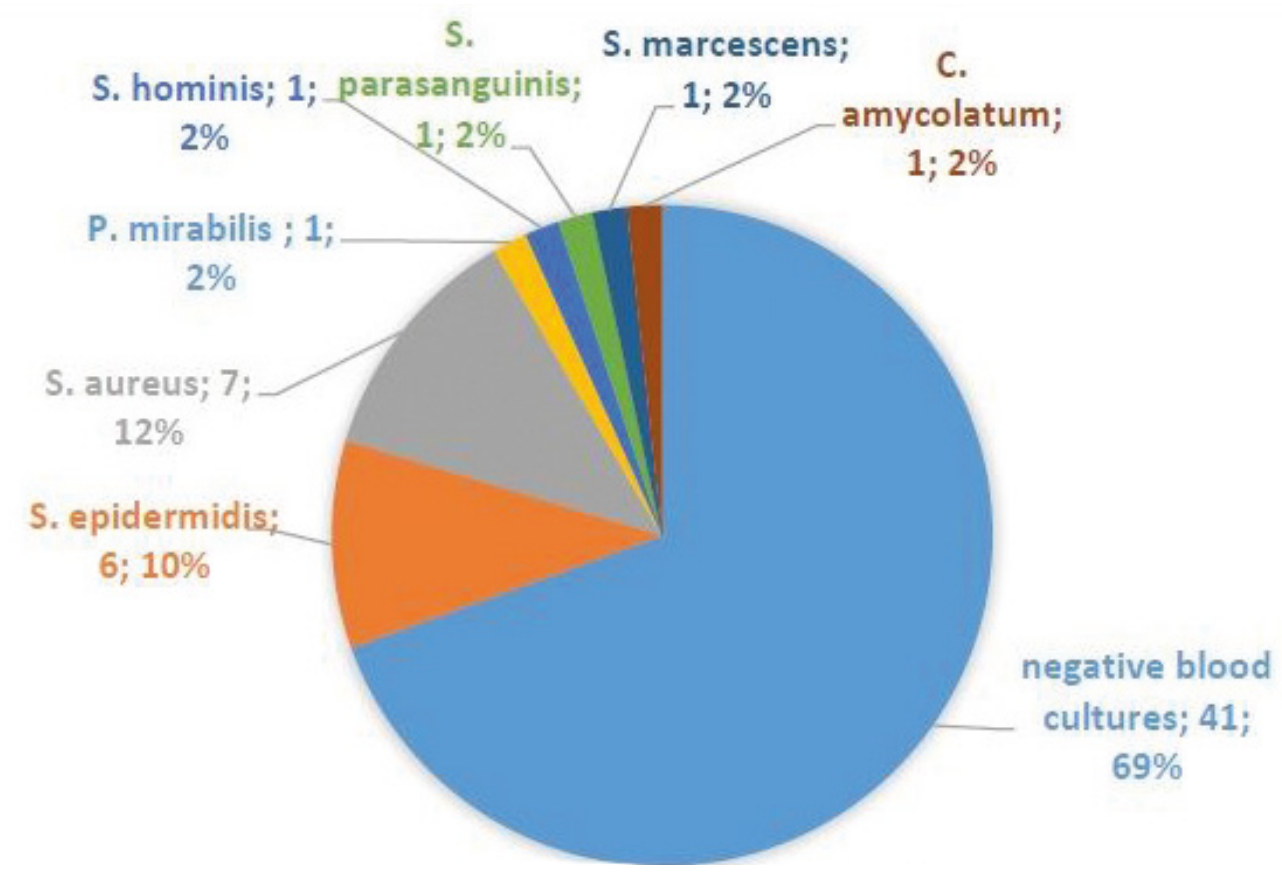

Figure 2. Etiologies of ICED infection based on blood cultures in the studied sample 
The timeframe between the most recent electrotherapy procedure and TLE due to ICED infection was 237 days (median; 25th and 75th percentile respectively 59 and 459 days). $56 \%$ ( $n=33$ ) of the analyzed sample had artificial cardiac pacemakers, while $44 \%$ had ICD (for details see Table 2). Details concerning severe ICED infection group and the rest of the studied sample are listed in Table 3.

Table 2. The most recent procedure preceding the TLE

\begin{tabular}{|lc|}
\multicolumn{1}{|c|}{ Procedure type: } & $\mathbf{n}(\%)$ \\
\hline - implantation de novo & $28(47.5)$ \\
- replacement & $15(25.4)$ \\
- upgrade & $16(27.1)$ \\
\hline Previous procedure: & \\
- device replacement & $19(32)$ \\
- device revision & $5(8.5)$ \\
- upgrade & $5(8.5)$ \\
\hline Pacemaker: & $33(56)$ \\
- AAI & $2(3.4)$ \\
- VVI & $6(10.2)$ \\
- DDD & $25(42.4)$ \\
- CRT-P & $0(0)$ \\
\hline Implantable cardioverter & $26(44)$ \\
defibrillator: & $12(20.3)$ \\
- ICD VR & $9(15.2)$ \\
- ICD DR & $5(8.5)$ \\
- CRT-D &
\end{tabular}

Abbreviations: CRT-P - cardiac resynchronization therapy pacemaker; CRT-D - cardiac resynchronization therapy defibrillator; ICD - implantable cardioverter defibrillator; TLE - transvenous lead extraction
Implantation de novo more frequently preceded TLE among the subset with severe ICED infection than among the rest of the analyzed sample ( $80 \%$ vs $40.8 \%, p=0.02$ ). Laboratory parameters from the time of the preceding electrotherapy procedure revealed median levels of: CRP $13.8 \pm 27.2 \mathrm{mg} / \mathrm{l}$, leukocytes $6.7 \pm 1.8 \times 10^{3} / \mu \mathrm{l}$, neutrocytes $4.3 \pm 1.7 \times 10^{3} / \mu \mathrm{l}$, creatinine $1.1 \pm 0.5 \mathrm{mg} / \mathrm{dl}$, hemoglobin $13.5 \pm 1.8 \mathrm{~g} / \mathrm{dl}$. The most frequent comorbidities among the studied sample are listed in Table 4. The median ejection fraction of the studied patients was $30 \%$. The median Charlson Comorbidity Index in the studied sample was 2 (see Figure 3 for details).

Table 4. Comorbidities among the sample

\begin{tabular}{|l|c|}
\hline Hypertension & $34(57.6 \%)$ \\
\hline Coronary artery disease & $30(50.8 \%)$ \\
\hline Chronic heart failure & $29(49.2 \%)$ \\
\hline Chronic atrial fibrillation & $17(28.8 \%)$ \\
\hline Diabetes mellitus & $13(22 \%)$ \\
\hline Paroxysmal atrial fibrillation & $12(20.3 \%)$ \\
\hline Chronic kidney disease & $5(8.5 \%)$ \\
\hline $\begin{array}{l}\text { Chronic obstructive pulmonary } \\
\text { disease }\end{array}$ & $5(8.5 \%)$ \\
\hline Mechanical heart valve & $5(8.5 \%)$ \\
\hline Neoplastic disease & $4(6.8 \%)$ \\
\hline
\end{tabular}

Table 3. Clinical characteristics of patients in severe ICED infection group and the rest of sample

\begin{tabular}{|c|c|c|c|}
\hline Variable & $\begin{array}{l}\text { Severe ICED } \\
\text { infection group } \\
(n=10)\end{array}$ & $\begin{array}{l}\text { Non-severe ICED } \\
\text { infection group } \\
(n=49)\end{array}$ & P value \\
\hline Median of age [years] & 70 & 74 & 0.09 \\
\hline $\begin{array}{l}\text { Device's type: } \\
\text { Pacemaker } \\
\text { ICD }\end{array}$ & $\begin{array}{l}6(60 \%) \\
4(40 \%)\end{array}$ & $\begin{array}{l}27(55 \%) \\
22(45 \%)\end{array}$ & 0.77 \\
\hline $\begin{array}{l}\text { Any previous electrotherapy } \\
\text { procedure: } \\
\text { Yes } \\
\text { No }\end{array}$ & $\begin{array}{l}8(80 \%) \\
2(20 \%) \\
\end{array}$ & $\begin{array}{l}20(41 \%) \\
29(59 \%) \\
\end{array}$ & 0.02 \\
\hline $\begin{array}{l}\text { Median of timeframe from last } \\
\text { procedure to TLE [days] }\end{array}$ & 407 & 151 & 0.25 \\
\hline $\begin{array}{l}\text { Infection's subtype: } \\
\text { PI } \\
\text { ICED-LI } \\
\text { ICED-IE }\end{array}$ & $\begin{array}{c}0(0 \%) \\
10(100 \%) \\
0(0 \%)\end{array}$ & $\begin{array}{c}36(73 \%) \\
10(20 \%) \\
3(7 \%) \\
\end{array}$ & $\begin{array}{l}<0.001 \\
<0.001 \\
0.39\end{array}$ \\
\hline $\begin{array}{l}\text { Positive blood cultures } \\
\text { Yes } \\
\text { No }\end{array}$ & $\begin{array}{l}5(50 \%) \\
5(50 \%)\end{array}$ & $\begin{array}{l}13(27 \%) \\
36(73 \%)\end{array}$ & 0.15 \\
\hline Charlson Comorbidity Index & 3 & 2 & 0.66 \\
\hline
\end{tabular}

Abbreviations: ICD - implantable cardioverter defibrillator; ICED - implantable cardiac electronic device; ICED-IE - implantable cardiac electronic device - infective endocarditis; ICED-LI -implantable cardiac electronic device - lead infection; PI - pocket infection; TLE - transvenous lead extraction 


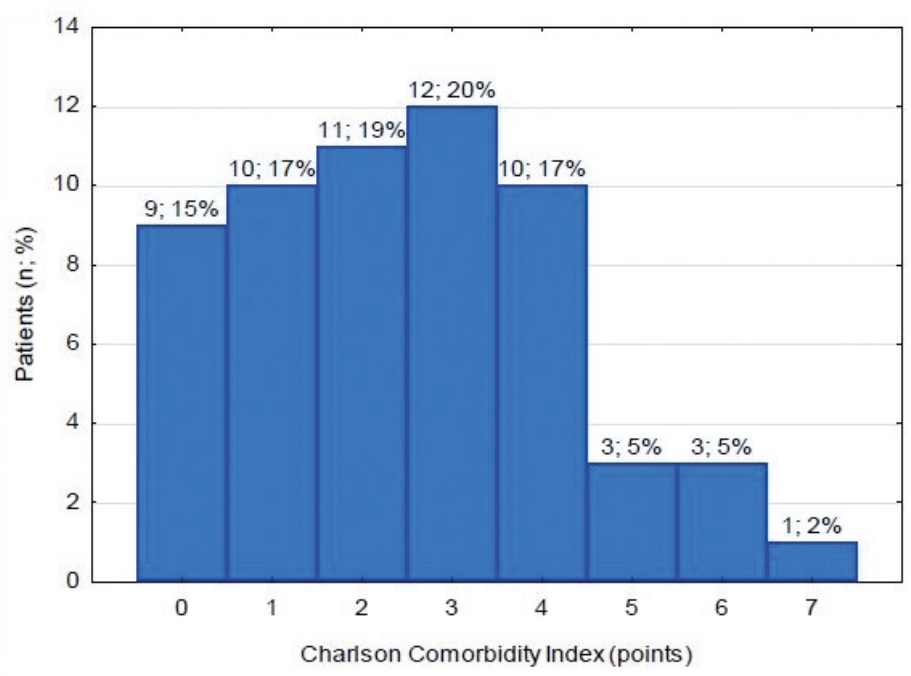

Figure 3. Charlson Comorbidity Index among the sample

Analysis of the pharmacotherapy administered in the pre-TLE period indicated that majority of the analyzed patients were on antiplatelet monotherapy $(n=19,32.2 \%)$ or anticoagulant monotherapy $(n=20,33.9 \%)$. In the anticoagulant group, $13(22 \%)$ patients were administered a vitamin $\mathrm{K}$ antagonist (VKA) and 7 (11.9\%) a non-VKA anticoagulant. The so-called bridge therapy using low molecular weight heparin (LMWH) was administered to 16 (27.1\%) patients, significantly more frequently among the patients with clinically severe ICED infections (60\% vs $20.4 \%$; $\mathrm{p}=0.04)$.

\section{Discussion}

In the recent years there was an increase in the number of implantations of implantable cardioverter-defibrillators (ICD) and pacemakers [4]. The ongoing increase in ICED implantations is associated with a concurrent increase in infectious complications [5]. It is estimated that $0.5-4.8 \%$ patients with ICED develop ICED infections, majority of which are PI [6-7]. In our sample, $61 \%$ of the patients underwent $\mathrm{PI}$ which is consistent with the findings in literature (58\% and 69\%) [6-7].

Our analysis was limited to patients, in whom a procedure of implantation, exchange or upgrade of the system had been identified within the preceding 2 years, because the aim of the study was to identify risk factors present at the time of the index procedure and leading to the development of infective complications. In our opinion if such a factor is present at the initial procedure, then its influence on the subsequent infection will reveal in a relatively short period of time, from several to a dozen or so months. Infections occurring later are - in our opinion - less likely to be associated with the in- itial procedure, and more likely result from infection-related factors during the following period.

The in-hospital mortality of ICED infections was higher in our sample than in literature ( $8.5 \%$ vs $4.1 \%$ ), which is due to a high subset of patients who developed septic shock [8].Majority of patients in our sample had implanted pacemakers. In the literature there are inconsistent findings as to which type of ICED is associated with a greater risk of infectious complications [9-12]. In our sample, we didn't find statistical difference between frequency of ICED infections in patients who had implanted pacemakers or implantable cardioverter-defibrillators. Interesting is the theory presented by Małecka at al. [13]. According to the author, the damage of the external insulation may result in infectious complications when the problem concerns pacemaker leads and to inappropriate interventions in the case of the damage of ICD leads. These differencies occur due to distinct construction these two types of leads.

Literature data provides that previous procedures such as revisions, device replacement and upgrades are well-documented risk factors of ICED infections [14]. Approximately half of the patients in our sample underwent a prior electrotherapy procedure, whereas those with severe ICED infections significantly more often had a device implantation de novo. This might be due to the fact that de novo implantations frequently are performed urgently, whereas device replacement and upgrade are planned procedures.

It is noteworthy that in our sample the patients undergoing TLE due to infection had on average three-fold increase in CRP level pre-operatively. This observation might confirm the earlier findings that patients with elevated CRP levels have a higher risk of developing ICED infections [15]. However this observation needs to be corroborated in a prospective study on a larger sample. 
Patients in our sample had a relatively high comorbidity as measured by the Charlson Index: $28.8 \%$ scored $\geq 4$ points. Charlson Comorbidity Index is considered a new risk factor of ICED infections. Our study is one of a few [16] confirming the potential correlation between Charlson Index value and ICED infection risk. The LMWH bridge therapy was administered rather frequently in the analyzed sample (27.1\%), particularly the patients who developed serious ICED infection. Literature points out that LMWH bridge therapy is an independent risk factor of device pocket hematomas, which in turn are a risk factor of ICED infections [17].

\section{Limitations}

Our study is limited due to small patient sample and the retrospective analysis. Therefore, our results are strictly preliminary and require confirmation in a prospective study with a larger sample. In addition, the electrotherapy procedures prior to TLE were conducted at various institutions, which might influence the results.

\section{Conclusions}

In the analyzed sample, the most common infectious complication related to the ICED was PI and the most common etiological agents were $S$. aureus and S. epidermidis. Patients with multiple comorbidities are often at risk of infectious complications and therefore require interdisciplinary care. Severe ICD infections that present with septic shock are associated with a $50 \%$ in-hospital mortality rate.

\section{References}

1. Sandoe JAT, Barlow G, Chambers JB, Gammage M, Guleri A, Howard P, et al. Guidelines for the diagnosis, prevention and management of implantable cardiac electronic device infection. Report of a joint Working Party project on behalf of the British Society for Antimicrobial Chemotherapy (BSAC, host organization), British Heart Rhythm Society (BHRS), British Cardiovascular Society (BCS), British Heart Valve Society (BHVS) and British Society for Echocardiography (BSE). J Antimicrob Chemother. 2015;70(2):325-359.

2. Singer M, Deutschman CS, Seymour CW, Shankar-Hari M, Annane D, Bauer M, et al. The third international consensus definitions for sepsis and septic shock (sepsis-3). Jama. 2016;315(8):801-810.

3. Charlson ME, Pompei P, Ales KL, MacKenzie CR. A new method of classifying prognostic comorbidity in longitudinal studies: development and validation. J Chronic Dis. 1987;40(5):373-383.

4. Tarakji KG, Ellis CR, Defaye P, Kennergren C. Cardiac implantable electronic device infection in patients at risk. Arrhythmia Electrophysiol Rev. 2016;5(1):65.

5. Baddour LM, Epstein AE, Erickson CC, Knight BP, Levison ME, Lockhart PB, et al. Update on cardiovascular implantable electronic device infections and their management: a scientific statement from the American Heart Association. Circulation. 2010;121(3):458-477.

6. Ann HW, Ahn JY, Jeon YD, Jung IY, Jeong SJ, Joung B, et al. Incidence of and risk factors for infectious complications in patients with cardiac device implantation. Int J Infect Dis. 2015;36:9-14.

7. Sohail MR, Uslan DZ, Khan AH, Friedman PA, Hayes DL, Wilson WR, et al. Management and outcome of permanent pacemaker and implantable cardioverter-defibrillator infections. J Am Coll Cardiol. 2007;49(18):1851-1859.

8. Deharo J-C, Quatre A, Mancini J, Khairy P, Le Dolley Y, Casalta J-P, et al. Long-term outcomes following infection of cardiac implantable electronic devices: a prospective matched cohort study. Heart. 2012;98(9):724-731.

9. Uslan DZ, Sohail MR, Sauver JLS, Friedman PA, Hayes DL, Stoner SM, et al. Permanent pacemaker and implantable cardioverter defibrillator infection: a population-based study. Arch Intern Med. 2007;167(7):669-675.

10. Voigt A, Shalaby A, Saba S. Rising rates of cardiac rhythm management device infections in the United States: 1996 through 2003 . J Am Coll Cardiol. 2006;48(3):590-591.

11. Nakajima H, Taki M. Incidence of cardiac implantable electronic device infections and migrations in Japan: results from a 129 institute survey. J arrhythmia. 2016;32(4):303-307.

12. Lekkerkerker JC, van Nieuwkoop C, Trines SA, van der Bom JG, Bernards A, van de Velde ET, et al. Risk factors and time delay associated with cardiac device infections: Leiden device registry. Heart. 2009 May 1;95(9):715-720.

13. Małecka B, Ząbek A, Ciaś A, Stępiński J, Kutarski A, Rońda J, et al. Endocardial silicone lead wear: description of tribological phenomena on the basis of microscopic examination of removed leads. Preliminary report. Kardiol Pol (Polish Hear Journal). 2014;72(10):960-968.

14. Polyzos KA, Konstantelias AA, Falagas ME. Risk factors for cardiac implantable electronic device infection: a systematic review and meta-analysis. Europace. 2015;17(5):767-777.

15. Sławiński G, Kempa M, Lewicka E, Budrejko S, Królak T, Raczak G. Elevated Creactive protein levels during cardiac implantations may increase the risk of early complications requiring transvenous lead removal: a preliminary report. Polish Arch Intern Med. 2018;128(2):138-140.

16. Sohail MR, Hussain S, Le KY, Dib C, Lohse CM, Friedman PA, et al. Risk factors associated with early-versus late-onset implantable cardioverter-defibrillator infections. J Interv Card Electrophysiol. 2011;31(2):171-183.

17. Ahmed I, Gertner E, Nelson WB, House CM, Dahiya R, Anderson CP, et al. Continuing warfarin therapy is superior to interrupting warfarin with or without bridging anticoagulation therapy in patients undergoing pacemaker and defibrillator implantation. Hear Rhythm. 2010;7(6):745-749. 